\title{
O USO DA MOULAGE NA SIMULAÇÃO CLÍNICA: ESTUDO DE CASOS MÚLTIPLOS
}

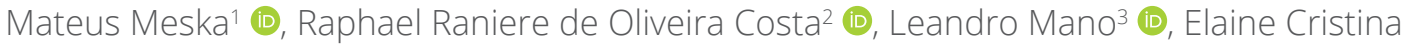 \\ Negri Santos ${ }^{4}$ (D), Barbara Casarin Henrique-Sanches, ${ }^{1, *}$ (D), Alessandra Mazzo5 ${ }^{\text {(i) }}$
}

\section{RESUMO}

Objetivo: identificar como e porque utilizar a moulage dentro das práticas de simulação clínica. Método: trata-se de estudo de casos múltiplos, inclusivo, de caráter descritivo e exploratório com abordagem qualitativa. Os casos foram selecionados por meio de uma amostra intencional. Após aplicação dos critérios de elegibilidade, foram incluídos e analisados quatro casos de cenários simulados que utilizaram moulage. Resultados: todos os casos analisados foram desenvolvidos em atividades clínicas simuladas extracurriculares por facilitadores com experiência em práticas clínicas, na docência e na área de simulação. As moulages utilizaram técnicas diferentes, promovendo maior interação com o aprendiz pelo uso de estimulantes de diferentes órgãos do sentido, aguçando o raciocínio clínico, o desenvolvimento de habilidades, competências, entre outros. Nos quatro casos, a moulage foi realizada em paciente simulado, em cenários simulados e vídeo simulado. Os motivos descritos para moulage foram: prover realismo, fidelidade, fácil aplicação e baixo custo. Conclusão: foi possível identificar que a moulage é um recurso adequando em simulação clínica e que quando construída com rigor e método, influencia na fidelidade e no realismo do cenário simulado.

DESCRITORES: Modelos anatômicos. Simulação. Educação em saúde. Educação superior. Estomaterapia.

\section{THE USE OF MOULAGE IN CLINICAL SIMULATION: MULTIPLE CASE STUDIES}

\begin{abstract}
Objective: identify how and why to use moulage within clinical simulation practices. Method: it is a multiple, inclusive, descriptive and exploratory case study with a qualitative approach. The cases were selected through an intentional sample. After applying the eligibility criteria, four cases of simulated scenarios that used moulage were included and analyzed. Results: all the cases analyzed were developed in extracurricular simulated clinical activities by facilitators with experience in clinical practices, teaching and simulation. The moulages used different techniques, promoting greater interaction with the learner through the use of stimulants of different sense organs, sharpening clinical reasoning, the development of skills, competences, among others. In the four cases, the moulage was performed on a simulated patient, in simulated scenarios and simulated video. The reasons described for moulage were: to provide realism, fidelity, easy application and low cost. Conclusion: it was possible to identify that the moulage is an adequate resource in clinical simulation and that when built with rigor and method, it influences the fidelity and realism of the simulated scenario.
\end{abstract}

DESCRIPTORS: Anatomical models. Simulation. Health education. College education. Stomatherapy.

1. Universidade de São Paulo - Escola de Enfermagem - Ribeirão Preto (SP), Brasil.

2. Universidade Federal do Rio Grande do Norte - Escola Multicampi de Ciências Médicas - Caicó (RN), Brasil.

3. Universidade Estadual do Rio de Janeiro - Telemedicina e Telessaúde - Rio de Janeiro (RJ), Brasil.

4. Universidade do Oeste Paulista - Departamento de Enfermagem - Presidente Prudente (SP), Brasil.

5. Universidade de São Paulo - Faculdade de Odontologia - Bauru (SP), Brasil.

*Autora correspondente: barbara.henrique@gmail.com

Editor de Seção: Juliano Teixeira Moraes

Recebido: Out. 19, 2021 | Aceito: Dez. 15, 2021

Como citar: Meska M; Costa RRO; Mano L; Santos ECN; Henrique-Sanches BC; Mazzo A. O uso da moulage na simulação clínica: estudo de casos múltiplos. ESTIMA, Braz. J. Enterostomal Ther., 2021, 19: e2921. https://doi.org/10.30886/estima.v19.1141_PT 


\section{EL USO DEL MOULAGE EN LA SIMULACIÓN CLÍNICA: ESTUDIO DE CASOS MÚLTIPLES}

\section{RESUMEN}

Objetivo: identificar cómo y por qué utilizar el moulage dentro de las prácticas de simulación clínica. Método: se trata de estudio de casos múltiples, inclusivo, de carácter descriptivo y exploratorio con abordaje cualitativo. Los casos fueron seleccionados por medio de una muestra intencional. Luego de aplicar los criterios de elegibilidad, se incluyeron y analizaron cuatro casos de escenarios simulados que utilizaron moulage. Resultados: todos los casos analizados fueron desarrollados en actividades clínicas simuladas extracurriculares por facilitadores con experiencia en prácticas clínicas, en la docencia y en el área de simulación. Los moulages utilizaron técnicas diferentes, favoreciendo una mayor interacción con el aprendiz por el uso de estimulantes de diferentes órganos del sentido, aguzando el raciocinio clínico, el desarrollo de habilidades, capacidades, entre otros. En los cuatro casos, el moulage se realizó en paciente simulado, en escenarios simulados y video simulado. Los motivos descritos para el moulage fueron: proveer realismo, fidelidad, fácil aplicación y bajo costo. Conclusión: fue posible identificar que el moulage es un recurso adecuado en simulación clínica y que cuando se construye con rigurosidad y método, influye en la fidelidad y en el realismo del escenario simulado.

DESCRIPTORES: Modelos anatómicos. Simulación. Educación para la salud. Educación superior. Estomaterapia.

\section{INTRODUÇÃO}

$\mathrm{Na}$ simulação clínica, cada vez mais os docentes e instrutores têm investido em maior nível de fidelidade, em cenários simulados, com o objetivo de proporcionar ao aprendiz experiências clínicas simuladas que se aproximem o mais próximo possível do real, o que exclui o "fazer de conta" e incorpora a criação e / ou replicação de ambientes ricos e atrativos, com o objetivo de motivar - de forma direta - o aprendiz, tornando a aprendizagem mais significativa ${ }^{1}$. Sabe-se que quanto mais real é a simulação, maior é o nível de aprendizagem e maior é o nível de satisfação dos estudantes ${ }^{2}$.

A fidelidade de um cenário simulado é frequentemente avaliada por meio de face e conteúdo. Quão realista se faz o cenário simulado o valida em face. Já a validade do conteúdo observa a sua adequação aos objetivos de aprendizagem ${ }^{3,4}$. A fidelidade das práticas simuladas tem sido equiparada ao quanto essas atividades podem imitar a realidade clínica, sendo, inclusive, classificadas como de alta, média e / ou de baixa fidelidade ${ }^{4,5}$, baseando-se, geralmente, na complexidade da simulação.

O conceito de fidelidade associa-se cada vez mais ao realismo, considerando as dimensões físicas, conceituais e atitudinais ${ }^{1}$. A dimensão física pode variar de baixa a alta fidelidade, de acordo com a tecnologia do simulador e o montante que o ambiente replica o ambiente real, incorporando a presença de sons, cheiros e adereços que representam a situação clínica. É nessa dimensão de realismo que a moulage se insere, uma vez que dispõe de recursos que podem conferir características visuais, de texturas e odores, os quais podem contribuir para aguçar emoções, sentimentos, raciocínio clínico e demais habilidades a serem desenvolvidas nos estudantes.

A definição de moulage associa-se ao uso de maquiagem de efeitos especiais, que simulam doenças, contusões, feridas, sangue, incisões, hematomas, idade do paciente, características clínicas e / ou outros efeitos a um manequim / simulador ou paciente simulado ${ }^{6}$. Essa adição de efeitos especiais, em simuladores e ou pacientes simulados, fornece pistas que geralmente não deveriam ser fornecidas de forma passiva, através da leitura do caso clínico e ou da informação fornecida pelo facilitador durante o pré-briefing ou briefing do cenário, o que permite aos facilitadores preencherem a lacuna que geralmente ocorre entre um caso clínico real e um caso clínico simulado?

Os benefícios do uso da moulage na simulação clínica são discutidos na literatura científica. Diversos estudos discutem seu potencial como ferramenta que possibilita aumentar a confiança dos profissionais nos mais diversos cenários, indicando a presença de queimaduras, melanomas, entre muitos outros sinais passíveis de avaliação num exame clínico ${ }^{8}$.

Quando usada apropriadamente, a moulage auxilia o aprendiz a confirmar os sinais clínicos que apoiam o diagnóstico. Outros estudos têm demonstrado, ainda, que o uso da moulage em simuladores ou pacientes simulados permite o treino de 
habilidades, como a estabilização de um trauma penetrante ${ }^{9,10}$, a realização da técnica de desbridamento em feridas ${ }^{5}$, a palpação e visualização de tecidos, a drenagem de abscesso simulado, entre muitos outros ${ }^{10}$. Além disso, aumenta o conhecimento, diminuindo o tempo de resposta para uma intervenção, estimula o pensamento crítico, o realismo, o engajamento sensorial, o que minimiza a descrença no realismo do cenário simulado?.

Apesar das pesquisas disponíveis na literatura científica, ainda não é possível encontrar teorias que orientem os níveis de fidelidade da moulage para o uso em simulação. Nesse contexto, sugerimos que no cenário clínico simulado, a moulage possa ser avaliada e classificada em Moulage de Baixa, Média e Alta Fidelidade, considerando, sobretudo, o realismo proporcionado por meio de estímulo visual, estético, tátil, de produção de odores, de número de pistas proporcionadas ao raciocínio clínico e de oportunidade de treino de habilidades.

A Moulage de Baixa Fidelidade, não menos importante, mas executada com poucos recursos materiais, realiza-se de forma mais simplista, bidimensional (2D), apresentando largura e comprimento. Tem o intuito de fornecer pistas para o desenvolvimento do cenário simulado, como hematomas, icterícia, palidez, escoriações, sudorese, sangramentos, diversos tipos de lesões, caracterização de paciente idoso, dentre outras ${ }^{11}$.

A Moulage de Média Fidelidade utiliza técnicas e recursos materiais com maior incremento para sua confecção. Apresenta-se na forma tridimensional (3D), com largura comprimento e altura ${ }^{12}$. Pode ainda incluir o uso de próteses de silicone 3D prémoldadas ${ }^{13}$ ou artefatos que os imitem, como ossos de galinha em uma fratura exposta. Fornece mais informações e / ou pistas para o desenvolvimento do cenário, como lesões abertas, lesões por pressão, úlcera venosa, cortes com sangramento, traumas, fraturas expostas, o que leva o aprendiz à necessidade de avaliar e refletir para que possa tomar decisões.

Já a Moulage de Alta Fidelidade é aquela que fornece estímulos aos sentidos dos estudantes, como o tato e o olfato. Pode conter formato 3D, assim como o acréscimo de outros estímulos multissensoriais e quadrimensionais (4D). Podem ter um custo mais elevado na sua confecção e requerem um tempo de maior de preparação. A definição de 4D é tida como recurso que objetiva imergir o telespectador na experiência, utilizando vibrações, movimentos, odores, e demais impulsos para outros órgãos do sentido ${ }^{14}$. Ao conferir essas características, a moulage 4D possibilita maior interação do estudante com o cenário clínico simulado e pode ser exemplificada por meio da análise de odor numa lesão confeccionada, por exemplo, com queijo provolone. Lesões com leito de ferida que possibilitam desbridamento, drenagem de abscessos, escarotomia, entre outros ${ }^{5,10,15}$.

Diferente dos simuladores que necessitam do uso de tecnologias robóticas para lhe conferirem nível de fidelidade, a moulage incorpora nos diferentes níveis de complexidade aqui propostos, a criatividade, o uso de materiais alternativos e diferentes técnicas para sua confecção e dependendo dos objetivos de aprendizagem traçados, quando associadas a simuladores e / ou pacientes simulados podem levar a um desfecho bastante favorável.

Nesse sentido este estudo teve por objetivo identificar como e porque utilizar a moulage dentro das práticas de simulação clínica.

\section{MÉTODO}

Trata-se de estudo de casos múltiplos ${ }^{16}$, inclusivo, de caráter descritivo e exploratório com abordagem qualitativa. Utilizou-se a seguinte questão de pesquisa: "Como e porque utilizar a moulage dentro das práticas de simulação clínica?"

Para tanto, fez-se a seguinte proposição: “A moulage quando construída com rigor e método, e em concordância com o cenário simulado, influência na avaliação clínica do paciente - por parte dos estudantes da área da saúde - e, consequentemente, no realismo da simulação" (estímulo visual, estético, tátil, de produção de odores, de número de pistas proporcionadas ao raciocínio clínico e de oportunidade de treino de habilidades).

Os casos foram selecionados por meio de uma amostra intencional, uma vez que se tratava de casos típicos ou especiais, estudados a partir de contextos reais. Para a coleta de dados foram, então, convidados - via e-mail - pela equipe da pesquisa, os experts da área de educação em saúde ou experts em simulação, que desenvolveram os casos simulados. O objetivo do contato foi apresentar os objetivos da pesquisa e esclarecer sobre as etapas seguintes. Em anexo, receberam o Termo de Consentimento Livre e Esclarecido (TCLE) e o Termo de autorização das imagens para fins de pesquisa. Após o aceite e 
preenchimento dos Termos, os experts foram direcionados a uma página online do Google Forms em que responderam a um questionário pré-estabelecido, em relação ao cenário simulado que desenvolveram utilizando moulage.

$\mathrm{O}$ questionário foi criado com base em Fabri ${ }^{17}$ e destaca os seguintes elementos: os objetivos do cenário; o públicoalvo; o método de ensino; os tipos de simuladores utilizados; a descrição do caso clínico; a descrição da moulage; o motivo da escolha da moulage; os recursos humanos (responsável pela confecção da moulage); o tempo de preparo da moulage; os recursos materiais e custos. Além disso, foi solicitado aos experts para anexarem as fotos da moulage confeccionada. Foi esclarecido, ainda, que a foto deveria ser recortada, com o objetivo de dar ênfase apenas à moulage e conter características de cor e resolução. Foi estipulado 20 dias de prazo para o aceite das respostas. De posse do instrumento preenchido e enviado pelos pesquisados, procedeu-se a análise dos dados.

Após busca e seleção de arquivos dos pesquisadores e dos casos de cenários simulados que utilizaram moulage, foram selecionados seis casos que ocorreram em diferentes contextos de ensino-aprendizagem (cenários simulados e vídeos simulados), a saber: 1) Assistência interprofissional às urgências respiratórias: cuidados com traqueostomia; 2) Assistência de Enfermagem ao paciente com ferida; 3) Trauma de extremidades; 4) Avaliação de feridas de hanseníase; 5) E-book de avaliação da lesão por pressão; 6) Assistência de Enfermagem ao paciente com ferida oncológica.

Para a análise do questionário enviado aos experts foi elaborado, pelos pesquisadores, com base nos mesmos critérios anteriores, um protocolo intitulado "Protocolo de análise de dados da Moulage em cenário clínico simulado", conforme demonstrado na Fig. $1^{18}$.

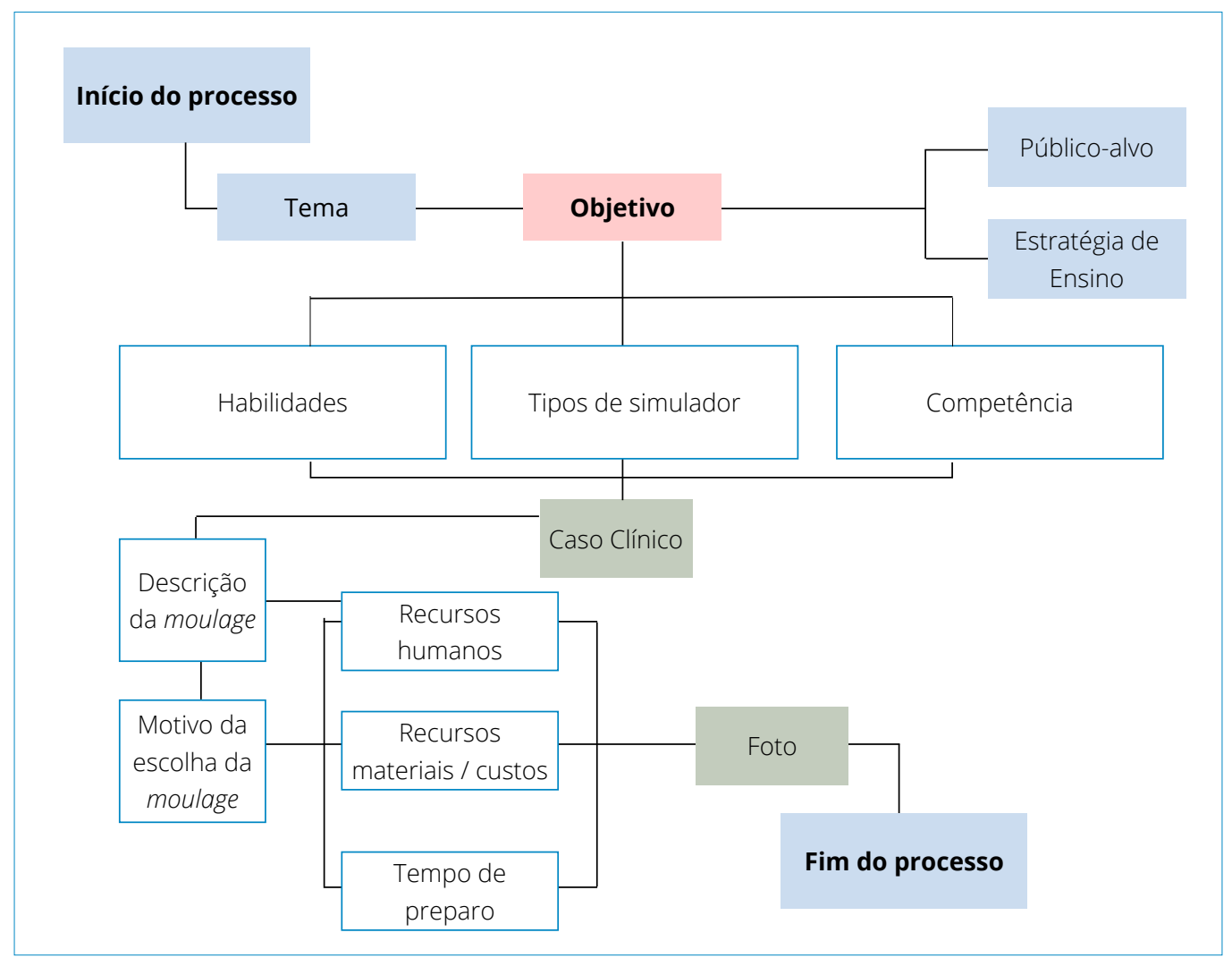

Figura 1. Protocolo de análise de dados da Moulage em cenário clínico simulado, elaborado pelos pesquisadores. Ribeirão Preto (SP) - 2019.

Após conferência e análise inicial das respostas contidas no formulário, foram incluídos no estudo os casos encaminhados pelos experts que continham fotos e descrição da moulage, seguindo as exigências elencadas pelos pesquisadores e o questionário respondido plenamente e no tempo determinado. Foram excluídos questionários preenchidos de forma incorreta e os casos em que as fotos continham logotipo de empresas, ou que pudessem identificar o paciente simulado ou marca do simulador. Posteriormente à aplicação dos critérios de elegibilidade, foram analisados quatro casos. 
Os dados foram analisados por meio dos itens das etapas da construção do cenário simulado e por meio da descrição e da foto da moulage confeccionada em cada caso. Os casos foram apresentados na forma de fluxograma, quadros e textos discursivos para melhor análise e comparação. Após essa etapa, foram analisados seguindo as proposições teóricas e os objetivos do estudo com base na revisão de literatura. Inicialmente, foram analisados de forma individual, e posteriormente foi realizado o cruzamento em sua totalidade ${ }^{16}$.

Este estudo teve aprovação do Comitê de Ética em Pesquisa da Escola de Enfermagem de Ribeirão Preto da Universidade de São Paulo, sob Parecer CAAE no 21241219.5.0000.5393.

\section{RESULTADOS}

Os quatro casos analisados foram desenvolvidos em atividades clínicas simuladas extracurriculares, por facilitadores com experiência em práticas clínicas, na docência e na área de simulação: caso 1) Assistência interprofissional em emergência respiratória: cuidados com traqueostomia; caso 2) Assistência de Enfermagem ao paciente com ferida; caso 3) Avaliação de feridas em hanseníase; caso 4) Trauma de extremidade ${ }^{18}$.

Todos os casos foram estruturados no fluxograma do protocolo de análise de dados da moulage em cenário clínico simulado, elaborado pelos pesquisadores.

Tabela 1. Casos simulados. Ribeirão Preto (SP) - 2021.

\begin{tabular}{|c|c|}
\hline Caso 1 & $\begin{array}{l}\text { ME.H.S., masculino, } 48 \text { anos, portador de câncer de laringe, em ar ambiente (via traqueostomia metálica), } \\
\text { está internado em pós-operatório de laringectomia total, Durante exame físico de enfermagem, paciente } \\
\text { começa a apresentar sinais de desconforto respiratório, com ruídos adventícios (tipo ronco) na ausculta } \\
\text { pulmonar. Se, porventura, for realizada aspiração de vias aéreas será encontrada resistência local. } \\
\text { Saturação deve cair, após aspiração, para 83\% e paciente mostrar cianose em extremidades. Se acionados } \\
\text { outros profissionais (fisioterapeuta e / ou médico) e oferecido, por um dos profissionais, oxigênio } \\
\text { suplementar, a saturação deve subir para 90\%. Após raciocínio, a equipe deve optar pela troca da cânula } \\
\text { interna de traqueostomia, após a troca de cânula a saturação deve se normalizar (97\%), assim como } \\
\text { quadro geral do paciente. }\end{array}$ \\
\hline Objetivo & Realizar troca de cânula da traqueostomia metálica. \\
\hline $\begin{array}{l}\text { Descrição da } \\
\text { Moulage }\end{array}$ & $\begin{array}{l}\text { Moulage em região cervical anterior do paciente simulado com presença de cânula de traqueostomia } \\
\text { metálica inserida / acoplada à moulage. }\end{array}$ \\
\hline Caso 2 & $\begin{array}{l}\text { M. S., } 62 \text { anos, sexo feminino, professora de ensino fundamental, deu entrada na enfermaria da clínica } \\
\text { cirúrgica do Hospital Geral, ontem, às } 10 \text { h da manhã. Apresenta lesão em membro inferior direito, em } \\
\text { região maleolar. Refere ser fumante há mais de } 20 \text { anos, hipertensa, tem varizes em membros inferiores. } \\
\text { Não faz atividade física por não ter tempo, e agora menos ainda, pois sente dor e tem medo de "machucar" } \\
\text { a ferida. Faz uso de Captopril } 25 \mathrm{mg} \text { e Hidroclorotiazida } 25 \mathrm{mg} 1 \text { vez / dia. A lesão apareceu há } 2 \text { anos, } \\
\text { e durante esse tempo vem tratando com papaína 4\%. Como não está tendo boa evolução, o médico } \\
\text { (vascular) decidiu interná-la para fazer alguns exames e iniciar tratamento com outra cobertura. Aguarda } \\
\text { avaliação do enfermeiro do setor. }\end{array}$ \\
\hline Objetivo & Identificar a úlcera crônica de etiologia vasculogênica por meio da consulta de enfermagem. \\
\hline $\begin{array}{l}\text { Descrição da } \\
\text { Moulage }\end{array}$ & $\begin{array}{l}\text { Nos membros inferiores do paciente simulado estão presentes alterações tróficas, edema, varizes, } \\
\text { hiperpigmetação, lipodermatoesclerose, eczema venoso, pulso periférico sem alteração. Na história clínica } \\
\text { representada, a dor é manifestada como intensa, no histórico torna-se mais forte ao final do dia e à noite. } \\
\text { o formato da lesão é irregular, extensa, exsudativa, há presença de grande quantidade de exsudato } \\
\text { amarelado, tecido de granulação, esfacelo e odor desagradável. }\end{array}$ \\
\hline
\end{tabular}


Tabela 1. Continuação...

\begin{tabular}{|c|c|}
\hline Caso 3 & $\begin{array}{l}\text { J.S., } 30 \text { anos, sexo masculino, produtor rural. Possui diagnóstico médico de hanseníase multibacilar. Em } \\
\text { tratamento regular com poliquimioterapia há seis meses. Não apresenta outras comorbidades. Procurou } \\
\text { serviço para administração da dose de medicação supervisionada mensal e para consulta de enfermagem. } \\
\text { Refere apresentar uma ferida na região plantar do pé direito e queixa-se de perder o chinelo ao caminhar, } \\
\text { sem se dar conta quando isso ocorre. Relata ainda andar descalço com frequência e usar botinas para } \\
\text { trabalhar. Ao ser indagado quanto ao cuidado com a ferida, refere lavar com água e sabonete durante o } \\
\text { banho e cobrir com gaze. Na avaliação neurológica dos membros inferiores, observa-se espessamento do } \\
\text { nervo fibular comum à direita e tibial posterior bilateral, com queixa de choque à palpação do nervo tibial } \\
\text { posterior à direita. Coloração de pele nos pés de cor típica, apresenta ressecamento, boa perfusão capilar, } \\
\text { pulsos normais, presença de calosidade e ausência de edema. Apresenta úlcera neuropática plantar (mal } \\
\text { perfurante plantar). }\end{array}$ \\
\hline Objetivo & Avaliar úlcera neuropática em pacientes com hanseníase. \\
\hline $\begin{array}{l}\text { Descrição da } \\
\text { Moulage }\end{array}$ & $\begin{array}{l}\text { Úlcera neuropática localizada na região plantar, com 2,3 cm de comprimento, tecido predominantemente } \\
\text { de granulação e bordas circulares com hiperqueratose ao redor. }\end{array}$ \\
\hline Caso 4 & $\begin{array}{l}\text { M.A.M., } 19 \text { anos, sexo masculino, motociclista, vítima de acidente de trânsito com colisão entre moto } \\
\text { e carro. No momento do acidente usava capacete. Foi trazido ao setor de emergência hospitalar por } \\
\text { populares. Encontra-se na maca, cabeceira a zero grau, ausência de prancha rígida e colar cervical, em } \\
\text { decúbito dorsal, respira espontaneamente em ar ambiente, abertura ocular espontânea, comunica-se } \\
\text { verbalmente e obedece a comandos. Apresenta escoriações por todo corpo. Ausência de ferimentos } \\
\text { profundos em tórax e abdome. Ausência de trauma cranioencefálico. Presença de fratura exposta em tíbia } \\
\text { direita. FC = } 110 \mathrm{bpm}, \mathrm{PA}=128 \times 86 \mathrm{mmHg} \text {, FR }=18 \mathrm{ipm} \text {, SO2 }=98 \% \text {, T = } 36.0^{\circ} \text {. }\end{array}$ \\
\hline Objetivo & Avaliar úlcera neuropática em pacientes com hanseníase. \\
\hline $\begin{array}{l}\text { Descrição da } \\
\text { Moulage }\end{array}$ & $\begin{array}{l}\text { O paciente simulado apresenta fratura exposta de tíbia direita com pequena quantidade de sangramento e } \\
\text { edema perilesional. }\end{array}$ \\
\hline
\end{tabular}

FC = Frequência cardíaca; bpm = Batimentos por minuto; PA = Pressão arterial; mmHg = milímetros de mercúrio; FR = Frequência respiratória; imp = Incursões por minuto; SO2 = Saturação de oxigênio; T = Temperatura.

Com base nos temas propostos, objetivos de aprendizagem e casos clínicos estipulados, os materiais de confecção da moulage foram definidos. Esses materiais tinham características de fácil acesso e eram de baixo custo, ficando restritos a látex profissional teatral $(80 \mathrm{~mL}-\mathrm{R} \$ 35,50)$, massa artística para efeitos especiais $(60 \mathrm{~g}-\mathrm{R} \$ 19,50)$, pigmentos de maquiagem vermelho, amarelo, roxo, marrom (10 g - R \$ 9,00 cada), sangue teatral artificial (120 mg - $\mathrm{R} \$ 32,90)$, pincel $(\mathrm{R} \$ 6,00)$, cano de PVC (1m x 20mm - R\$4,90), lixa de madeira ( $\left.\mathrm{n}^{\circ} 120-\mathrm{R} \$ 1,20\right)$, arco de serra $(R \$ 29,90)$, lima quadrada ( $\left.{ }^{\circ} 8-R \$ 21,90\right)$, kit com esponjas para maquiagem $(R \$ 4,90)$, agulha de crochê dupla $(\mathrm{R} \$ 2,90)$, além de um secador de cabelo de $1.000 \mathrm{~W}(\mathrm{R} \$ 34,90)$ e de restos alimentares fermentados que proporcionaram odor as lesões confeccionadas. As moulages foram confeccionadas por pós-graduando (caso 1, 2 e 3) e facilitador (caso 4) capacitados, e levaram de 40 minutos (casos 2 e 4) minutos a 2 horas (caso 1) para serem confeccionadas. A confecção da moulage do caso 3 levou 1 hora. Na Fig. 2 são demonstrados os resultados das moulages nos pacientes simulados.

Para demonstrar o uso do protocolo de avaliação proposto e utilizado pelos pesquisadores para descrição e análise de cada caso, a Fig. 3 apresenta as informações do caso 3) Avaliação de feridas em hanseníase. 


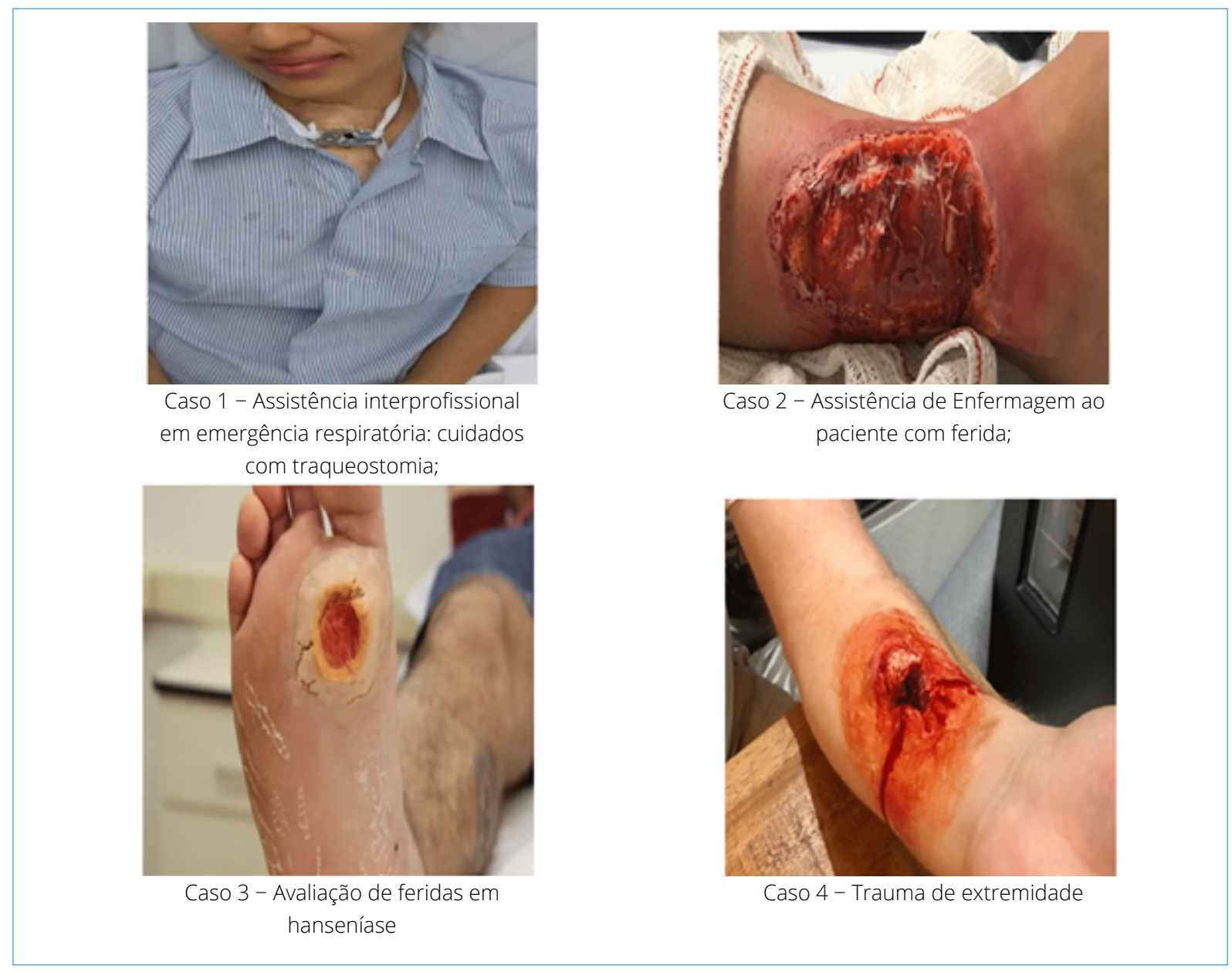

Figura 2. Resultado da confecção das moulages nos pacientes simulados dos casos 1, 2, 3 e 4 . Ribeirão Preto (SP) - 2020.

\section{DISCUSSÃO}

A diversificação de estratégias de ensino tem sido um componente fundamental dentro da organização curricular e da formação de profissionais de saúde ${ }^{2}$. No dinâmico processo de ensino-aprendizagem que se estabelece atualmente, os educadores devem utilizar recursos didáticos mais abrangentes e participativos, o que envolve entre muitas outras estratégias a construção de cenários clínicos simulados estruturados, com objetivos, habilidades e competências claras e bem definidas ${ }^{17}$.

A moulage pode ser utilizada em diferentes recursos educacionais, como vídeo educativo, cenário simulado, álbum seriado, cartilhas e / ou em outros, inclusive, quando há escassez de acervo fotográfico de imagens reais de estruturas, tecidos e condições clínicas. Nos casos analisados foi possível identificar o seu emprego na educação superior, no ensino de Enfermagem, Medicina e Fisioterapia, caracterizando pacientes e simuladores em atividades com objetivos de aprendizagem, competências e habilidades diversas, aproximando o aprendiz do campo clínico com realismo.

A fidelidade e autenticidade do cenário aprimoram o envolvimento dos participantes. A moulage amplia as possibilidades do simulador, permitindo o desenvolvimento de habilidades que extrapolam os padrões estáticos desses equipamentos. Essa associação, da moulage e do simulador, é ainda bastante útil em situações nas quais é necessário agregar a percepção sensorial do olfato, da visão ou outras (oferecidas por meio da moulage) a procedimentos invasivos, como a drenagem de um abcesso.

Em simulação, faz-se a prática de problemas clínicos vivenciados na prática clínica real, não somente usando a imaginação. Com o uso de moulage pode-se aproximar os cenários clínicos das características reais dos pacientes, fornecendo elementos aos aprendizes para identificarem, avaliarem, tomarem decisões e desenvolverem competências frente aos problemas encontrados. Nesse sentido, a moulage tem se mostrado como um recurso bastante utilizado para se trabalhar habilidades atitudinais e de 
comunicação com pacientes simulados. Todavia, quando utilizada em part task trainer ou simuladores de baixa fidelidade, a moulage possibilita o aumento de treino de habilidades com maior grau de dificuldade e, consequentemente, o realismo, como o emprego de odores e secreções em pelves femininas para a realização do exame especular.

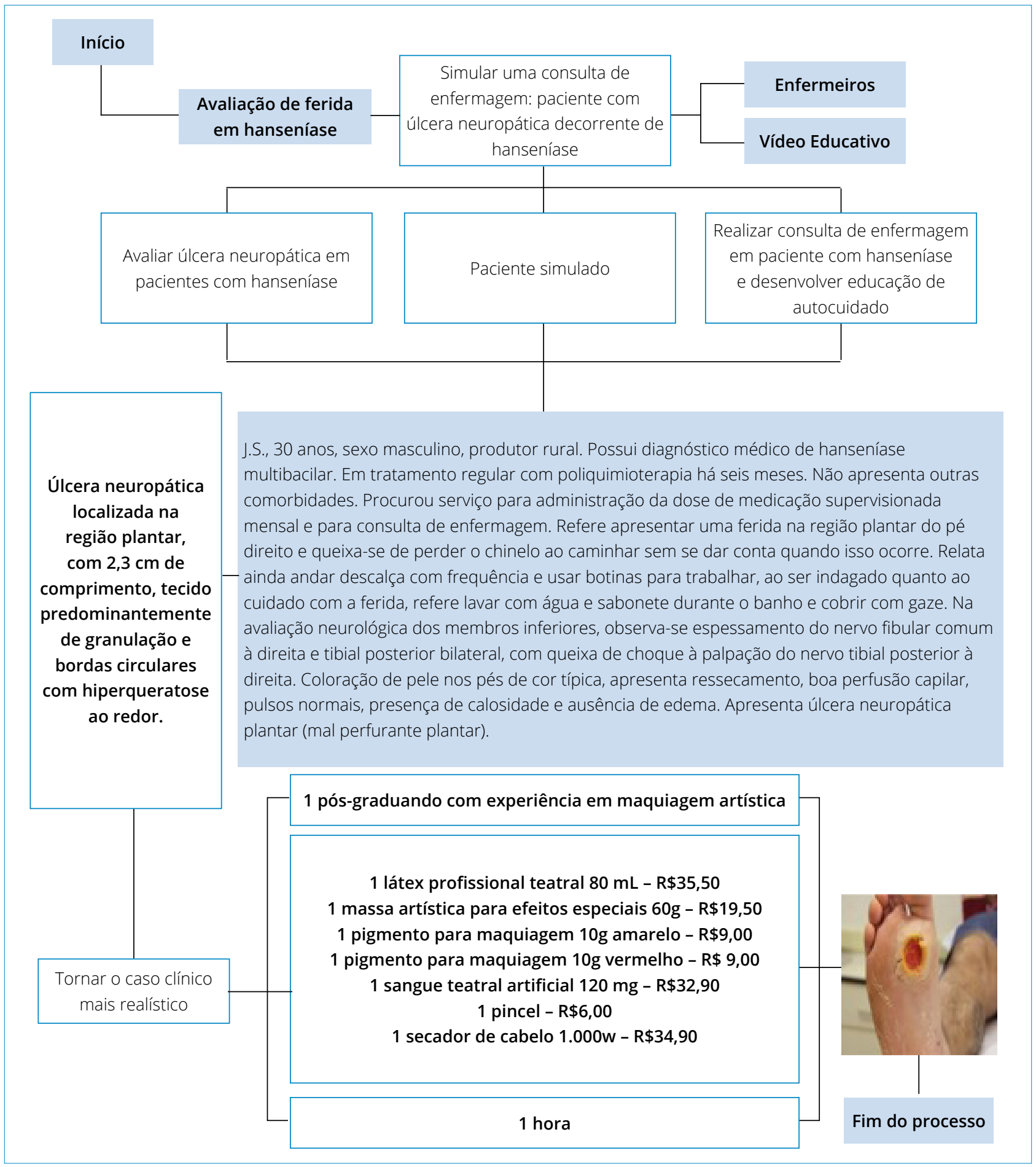

Figura 3. Protocolo de análise de moulage em cenário simulado do caso 3) Avaliação de ferida em hanseníase. Ribeirão Preto (SP) - 2020.

Ambientes e experiências clínicas simuladas devem ser o mais próximo possível do real. Expor aprendizes a cenários simulados que envolvam níveis mais altos de fidelidade, associados ao contexto legítimo, concretiza o aprendizado e tem efeito positivo na formação $0^{2,4,8,19,20}$. Além disso, cenários de alta fidelidade também promovem maiores scores de satisfação e autoconfiança na aprendizagem ${ }^{20}$. As moulages apresentadas nos estudos de caso analisados, além de prover fidelidade 
ao cenário simulado, foram capazes de fornecer elementos aos estudantes que auxiliaram a avaliação clínica e a tomada de decisões por meio de características visuais, táteis e olfatórias.

Durante a avaliação clínica, a observação é a etapa descrita como o uso atento dos sentidos (visão, audição, tato e olfato) para coletar atributos ou informações direcionadas às estruturas corporais, no plano geral e específico ${ }^{21}$. Em cenários simulados com o uso da moulage, o estudante pode aprimorar sua observação e, com isso, seus órgãos do sentido, identificando com a visão características como forma, área, relevo, simetria, cor, rede venosa, aspectos e condições da pele; com o tato, por meio da palpação, características, como tamanho, textura, formato, consistência e relevo; e com o olfato, odores característicos de processos fisiológicos e / ou odores desagradáveis, infecciosos exsudato, sangue, odor cetônico, entre outros ${ }^{18}$.

As pistas visuais em cenários clínicos simulados, conforme demonstram os casos de 1 a 4 , orientam continuamente o estudante durante a simulação, fornecendo feedback instantâneo e indicando que há algo a ser avaliado, interpretado e assistido, facilitando o raciocínio clínico e a tomada de decisão durante a simulação ${ }^{8}$. Ao serem preparadas com cores, texturas e odores e se tornarem moulages 2D, 3D e 4Ds, aumentam a sua fidelidade, surtindo maior precisão na avaliação clínica.

Os casos apontam que a moulage possibilita o treino de habilidades técnicas conforme exibido no caso 1 , em que foi confeccionado uma moulage 4D de alta fidelidade (cor, textura, altura, largura, que ainda possibilitou a realização da aspiração da traqueostomia e a análise do material aspirado) na região cervical, e com ela foi possível realizar a aspiração das vias aéreas e a troca da cânula de traqueostomia metálica, bem como foi ofertado no caso 4, moulage de fratura exposta, que proporcionou prática da estabilização do trauma. Nos casos 2 e 3 , embora os experts respondentes não tenham comentado na descrição do caso a realização do curativo, o uso de moulages de lesões pode servir para o treino de habilidades técnicas como higienização da lesão, avaliação do leito e tecidos presentes, realização de desbridamento, escolha de cobertura ideal, troca de curativo, entre outros ${ }^{15}$.

Os custos para seu emprego são variáveis. O docente e ou a instituição que pretendem fazer uso desse recurso podem ter gastos iniciais na aquisição de instrumentos específicos, como espátulas, moldes, paleta de sombras, látex, entre outros. Porém uma vez adquiridos, podem ser reutilizados em muitas atividades. Os custos variam de acordo com a extensão e características da maquiagem. Nos casos analisados, os custos variaram entre $\mathrm{R} \$ 75,54$ e $\mathrm{R} \$ 182,77$. Quando comparados os custos das moulages de silicone com o custo das moulages confeccionadas neste estudo, é possível observar que moulages que são confeccionadas com maquiagem apresentam valores mais acessíveis. Um dos benefícios da moulage de silicone é a não necessidade de uma pessoa habilitada para a sua confecção, uma vez que é adquirida no mercado e pode ser reutilizada diversas vezes, além de replicada em vários casos, o que a torna uma estratégia eficiente em treinos de habilidades e avaliações em que um mesmo cenário é repetido como nos Exames Clínicos Objetivos Estruturados.

O tempo de preparo das lesões, tecidos e estruturas é variável. Nos casos estudados, o tempo gasto descrito foi de 40 minutos para moulages menos complexa, e 2 horas para moulages mais complexa. $\mathrm{O}$ tempo também varia de acordo com a habilidade do maquiador. Em cenários de múltiplos casos é importante que mais de um maquiador desenvolva essa atividade. Profissionais de maquiagem aptos para confecção e execução da moulage são limitados no mercado. Além disso, os valores praticados para contratação de profissionais de maquiagem aptos para uso de moulage podem extrapolar os recursos de grande parte de programas de simulação ${ }^{22}$.

Os casos aqui descritos demonstraram que a construção de moulages não necessariamente exige profissionais, mas sim recurso humano capacitado, o que pode ser cumprido pelo próprio facilitador. Estudos anteriores minuciaram métodos de moulage de valor reduzido utilizando aparatos acessíveis aos profissionais de educação e descreveram como o facilitador pode executar moulage de baixo custo mimetizando lesões reais, ${ }^{23,24}$. Estudo recente descreveu que os estudantes que construíram suas próprias moulages as perceberam como bastante reais ${ }^{3}$. Há também experiências em que os estudantes aprendem e desenvolvem a moulage entre os pares.

\section{CONCLUSÃO}

Neste estudo de caso foi possível corroborar a proposição do estudo, uma vez que os casos demonstraram que a moulage, quando executada com rigor e método, acompanhando o cenário simulado, influencia na avaliação clínica dos estudantes durante a simulação e consequentemente no realismo da simulação clínica. 
Embora tenha sido realizado com quatro casos, o que alguns autores podem caracterizar como uma limitação, o estudo contribui para o avanço do conhecimento na medida em que analisa casos, compartilha um modelo / protocolo de análise de casos relacionados á moulage e aponta para uma discussão inicial sobre a categorização da moulage dentro do universo da simulação cínica.

Os autores encorajam docentes, instrutores e curiosos na área de simulação a incluir, a depender dos objetivos de aprendizagem, a moulage em experiências clínicas simuladas e a aferir as contribuições e impactos desses recursos em variáveis como o realismo, a satisfação do estudante e na aprendizagem. Além disso, a divulgação de novas experiências pode corroborar o desenvolvimento de estudos mais robustos e para o amplo uso dessa técnica de maquiagem no ensino em saúde e enfermagem.

\section{CONTRIBUIÇÃO DOS AUTORES}

Conceituação: Meska M e Mazzo A; Metodologia: Meska M, Costa RRO e Mazzo A; Investigação: Meska M, Mano L, Santos ECN e Mazzo A; Redação - Primeira versão: Meska M, Costa RRO, Mano L, Santos ECN, HenriqueSanches BC e Mazzo A; Redação - Revisão \& Edição: Meska M, Henrique-Sanches BC e Mazzo A; Recursos: Meska M; Supervisão: Mazzo M.

\section{DISPONIBILIDADE DE DADOS DE PESQUISA}

Não se aplica.

\section{FINANCIAMENTO}

Coordenação de Aperfeiçoamento de Pessoal de Nível Superior

Grant no.: Code 001

\section{AGRADECIMENTOS}

Ao Programa de Pós Graduação em Enfermagem Fundamental da Escola de Enfermagem de Ribeirão Preto da Universidade de São Paulo - EERP-USP.

\section{REFERÊNCIAS}

1. Lioce L, Meakim CH, Fey MK, Chmil JV, Mariani B, Alinier G. Standards of best practice: Simulation standard IX: Simulation design. Clin Simul Nurs. 2015;11(6):309-15. https://doi.org/10.1016/j.ecns.2015.03.005

2. Costa RRO, Medeiros SM, Martins JCA, Dias VR. Percepções de estudantes de enfermagem acerca das dimensões estruturais da simulação clínica. Sci med. 2019;29(1): e32792. https://doi.org/10.15448/1980-6108.2019.1.32972

3. Makkink AW, Slabber H. For the students, by the students: Student perceptions of low cost medical moulage in a resourceconstrained environment. Afr J Emerg Med. 2019;9(4):207-11. https://doi.org/10.1016/j.afjem.2019.08.003

4. Kaneko RMU, Lopes MHBM. Cenário em simulação realística em saúde: o que é relevante para a sua elaboração? Rev Esc Enferm USP. 2019;53:e03453. https://doi.org/10.1590/s1980- 220×2018015703453

5. Jeffries PR, Rogers KJ. Evaluating simulations. Simulation in nursing education: From conceptualization to evaluation. New York: National League for Nursing. 2007. 87-103.

6. Choong J, Tan ZY. The role of simulation in burns education. Br J Hosp Med. 2019; 80(12): 716-9. https://10.12968/ hmed.2019.80.12.716

7. Stokes-Parish JB, Duvivier R, Jolly B. Does appearance matter? Current issues and formulation of a research agenda for moulage in simulation. Simul Healthc. 2017;12(1):47-50. https://doi.org/10.1097/SIH.0000000000000211 
8. Mills BW, Miles AK, Phan T, Dykstra PMC, Hansen SS, Walsh AS et al. Investigating the extent realistic Moulage impacts on immersion and performance among undergraduate Paramedicine students in a simulation-based trauma scenario: a pilot study. Simul Healthc. 2018;13(5):331-40. https://doi.org/10.1097/SIH.0000000000000318

9. Stokes-Parish JB, Duvivier R, Jolly B. Investigating the impact of moulage on simulation engagement: a systematic review. Nurse Educ Today. 2018;(64):49-55. https://doi.org/10.1016/j. nedt.2018.01.003

10. Pai DR, Singh S. Medical simulation: overview, and application to wound modelling and management. Indian J Plast Surg. 2012;45(2):209-14. https://doi.org/10.4103/0970-0358.101280

11. Sezgunsay E, Basak T. Is Moulage effective in improving clinical skills of nursing students for the assessment of pressure injury? Nurse Educ Today. 2020;94:104572. https://doi.org/10.1016/j.nedt.2020.104572

12. Cazañas EF. Desvelando o uso da simulação nos cursos de graduação em enfermagem no Brasil. [tese]. Botucatu (SP): Faculdade de Medicina de Botucatu, Universidade Estadual Júlio de Mesquita Filho; 2020.

13. Garg A, Haley H, Hatem D. Modern moulage: evaluating the use of 3-dimensional prosthetic mimics in a dermatology teaching program for second-year medical students. Arch dermatol. 2010;146(2):143-6. https://doi.org/10.1001/archdermatol.2009.355

14. Silva DL. Além da tela: a experiência multissensorial das salas de cinema. Intercom. 2019;1-13. Disponível em: http:// portalintercom.org.br/anais/nacional2019/resumos/R14-0738-1.pdf

15. Mazzo A, Miranda FBG, Meska MHG, Bianchini A, Bernardes RM, Pereira Junior GA. Teaching of pressure injury prevention and treatment using simulation. Esc Anna Nery. 2018;22(1):e20170182. https://doi.org/10.1590/2177-9465-EAN-2017-0182

16. Yin RK. Estudo de caso: planejamento e métodos. $5^{\text {a }}$ ed. Porto Alegre: Bookman; 2015.

17. Fabri RP, Mazzo A, Martins JCA, Fonseca AS, Pedersoli CE, Miranda FBG et al. Construção de um roteiro teórico-prático para simulação clínica. Rev esc enferm USP. 2017;51:e03218. https://doi.org/10.1590/S1980-220X2016265103218

18. Meska MHG. O uso da moulage nas práticas de simulação clínica: estudo de casos múltiplos. [Dissertação]. Ribeirão Preto (SP): Escola de Enfermagem de Ribeirão Preto, Universidade de São Paulo; 2020.

19. Kim YJ, Noh GO, Im YS. Effect of step-based prebriefing activities on flow and clinical competency of nursing students in simulation-based education. Clin Simul Nurs. 2017;13(11):544-51. https://doi.org/10.1016/j.ecns.2017.06.005

20. Costa RRO, Medeiros SM, Coutinho VRD, Mazzo A, Araújo MS. Satisfacción y autoconfianza en el aprendizaje de estudiantes de enfermeria: Ensayo clínico aleatorizado. Esc Anna Nery. 2020;24(1):e20190094. https://doi.org/10.1590/2177-9465EAN-2019-0094

21. Bachion MM. Instrumentos básicos do cuidar: observação, interação e mensuração. Anais do I Simpósio Brasiliense de Sistematização da Assistência de Enfermagem de Brasília, 2009; Brasília. Disponível em: https://www.aben-df.com.br/ cursos_e_eventos/2010/simbrasae/INSTRUMENTOS_BASICOS_CUIDAR-MARIA_MARCIA_BACHION.PDF

22. Flores EK; Hess R. Comparing teaching methods on skin disorders using standardized patients dressed in moulage vs paper cases. Am J Pharm Educ. 2018;82(7):6636. https://doi.org/10.5688/ajpe6636

23. Sampson C, Parker CD, Burns E, Ruyan M, Higbee D, Koboldt T. (2018). A Low Cost Escharotomy Simulation Model for Residency Education. JETem. 2018;3(3):41-8. https://doi.org/10.5070/M533039877

24. Stokes-Parish J, Duvivier R, Jolly B. Expert opinions on the authenticity of moulage in simulation: a Delphi study. Adv Simul. 2019;4(1):1-10. https://doi.org/10.1186/s41077-019-0103-z 